\title{
Apnea Obstructiva del Sueño y el Rol del Ortodoncista. Revisión bibliográfica.
}

\section{Obstructive Sleep Apnea and the Role of the Orthodontist. Bibliographical review.}

\author{
1. Departamento de Ortodoncia, Facultad de \\ Odontología, Universidad de los Andes, Santiago, \\ Chile. \\ * Correspondencia Autor: Rodrigo Oyonarte. I \\ Dirección: Monseñor Álvaro del Portillo 12455, \\ Santiago, Las Condes, Región Metropolitana, Chile. \\ | E-mail: royonarte@miuandes.cl \\ Trabajo recibido el 04/05/2020 \\ Trabajo revisado 30/06/2020 \\ Aprobado para su publicación el 04/10/2020
}

María José Alvarado ${ }^{1}$, Rodrigo Oyonarte ${ }^{1 *}$

\begin{abstract}
RESUMEN
Introducción: La apnea obstructiva del sueño es un trastorno crónico, altamente prevalente, asociada a secuelas cardiovasculares y neurocognitivas, que afectan gravemente la calidad de vida de los individuos que la padecen. En aquel contexto, tanto el odontólogo general, como especialista, tienen un rol importante en el screening y potencial tratamiento interdisciplinario de esta patología. Revisión de la literatura actual: Se describen las causas y las secuelas asociadas a este trastorno, junto con los métodos de screening inicial que sirven para una derivación oportuna. Además, se plantean las alternativas de tratamiento y la evidencia actual disponible sobre sus efectos terapéuticos. Discusión y conclusión: Por ser la apnea obstructiva del sueño una patología compleja y de origen multifactorial, se aborda con un equipo interdisciplinario para determinar la mejor estrategia de tratamiento de acuerdo a las características de cada paciente, y entendiendo las repercusiones que cada terapia conlleva. En Ortodoncia, existen controversias respecto del efecto de los tratamientos con extracciones de premolares sobre las dimensiones de la vía aérea superior, donde es relevante analizar críticamente la evidencia científica disponible al respecto, y con ello, asegurar estrategias óptimas de tratamiento en la práctica clínica.
\end{abstract}

PALABRAS CLAVE:

Apnea obstructiva del sueño; ortodoncia; revisión.

Int. J. Inter. Dent Vol. 14(3); 242-245, 2021.

\section{ABSTRACT}

Introduction: Obstructive sleep apnea is a chronic, highly prevalent disorder associated with cardiovascular and neurocognitive repercussions, which seriously affect the quality of life of the individuals who suffer from it. In that context, both the general dentist and the specialist have an important role in the screening and potential interdisciplinary treatment of this disorder. Review of current literature: The causes and sequelae associated with this disorder are described, together with the initial screening methods that serve for a timely referral. In addition, treatment alternatives and current available evidence on their therapeutic effects are addressed. Discussion and conclusion: Since obstructive sleep apnea is a complex and multifactorial disorder, it is approached from an interdisciplinary perspective to determine the best treatment strategy according to the characteristics of the patient, with a clear understanding of the repercussions that each therapy involves. In Orthodontics, controversy exist about the effect of treatment with premolar extractions on the dimensions of the upper airway. It is relevant to critically analyze the available evidence about this controversy in order to ensure optimal treatment strategies in clinical practice.

KEY WORDS:

Sleep apnea; Obstructive; orthodontics; review.

Int. J. Inter. Dent Vol. 14(3); 242-245, 2021. 


\section{INTRODUCCIÓN}

La apnea obstructiva del sueño es un trastorno crónico, donde ocurre una obstrucción completa o parcial de la vía aérea al dormir, pudiendo acompañarse de somnolencia diurna excesiva, situación donde pasa a llamarse síndrome de apnea obstructiva del sueño (SAOS) ${ }^{(1)}$. Se encuentra asociada a las principales causas de muerte, como lo son la cardiopatía isquémica y el infarto cerebral ${ }^{(2,3)}$, además de asociarse a repercusiones cognitivas y afectar gravemente la calidad de vida de los individuos que la padecen ${ }^{(4)}$. En adultos tiene una prevalencia del 5 al $20 \%$, siendo el sexo masculino, un mayor índice de masa corporal (IMC) y una mayor edad, los principales factores de riesgo ${ }^{(5-8)}$. En niños, se presume que afecta entre el 1 al $3 \%$ de los preescolares, existiendo un peak entre los 2 y 6 años, coincidente con la etapa peak de hiperplasia linfoidea ${ }^{(9)}$.

Dada la alta prevalencia del SAOS, actualmente se constituye como un problema de salud pública, debido a la severidad de las secuelas asociadas. Se ha establecido una asociación entre SAOS y distintos cuadros perjudiciales para la salud, como trastornos cardiovasculares, la diabetes mellitus tipo 2 y el síndrome metabólico. Estas complicaciones pueden ser diagnosticadas tardíamente, siendo parte del curso y cronicidad del SAOS, en especial en sus grados moderados a severos ${ }^{(4,10,11)}$. No existe un entendimiento completo de los mecanismos patogénicos, pero es probable que sea multifactorial, incluyendo procesos de estrés oxidativo e inflamación sistémica(10).

A su vez, cabe recalcar que una secuela altamente relevante de SAOS es la alteración neurocognitiva y su impacto en la calidad de vida. La disfunción cognitiva se presenta con disminución en la atención, memoria y función ejecutiva, y puede llevar a somnolencia diurna excesiva, deterioro de la productividad en el trabajo, riesgo de accidentes automovilísticos, dolores de cabeza matutinos, alteraciones del estado de ánimo y trastornos del comportamiento neurológico ${ }^{(4,12,13)}$ De igual modo, la fragmentación del sueño e hipoxemia, específicamente en niños, altera su desarrollo físico y neurocognitivo, pudiendo generar problemas en la memoria, aprendizaje y rendimiento académico ${ }^{(13)}$.

Siendo el SAOS una patología compleja y altamente prevalente, tanto el odontólogo general como el odontólogo especialista, ya sea ortodoncista, cirujano maxilofacial, especialistas en trastornos temporomandibulares $u$ otros asociados a equipos de medicina del sueño, pueden cumplir roles fundamentales en su screening inicial, así como en su tratamiento. La siguiente revisión tiene por objetivo describir las causas y las secuelas asociadas a este trastorno, y discutir las alternativas terapéuticas disponibles y las controversias asociadas, con énfasis en la perspectiva ortodóncica en base a la evidencia disponible en la actualidad.

\section{REVISIÓN DE LITERATURA}

\section{Etiopatogenia}

La apnea obstructiva del sueño se produce por el colapso recurrente de la vía aérea superior al dormir, con la consiguiente obstrucción del flujo aéreo. Ocurre en sujetos que tienen alterada las propiedades mecánicas de su vía aérea, sumado a la reducción en la actividad del músculo geniogloso al inicio del sueño(10). A su vez, el ciclo del sueño de individuos con SAOS está caracterizado por despertares y la fragmentación del sueño con cada episodio de apnea ${ }^{(10)}$.

Distintos factores de riesgo pueden verse involucrados en pacientes adultos, entre los que se cuentan el sexo masculino, la mayor edad, menopausia, obesidad (IMC $\geq 30 \mathrm{~kg} / \mathrm{m}^{2}$ ), distribución central de grasa corporal, y uso de sedantes, entre otros ${ }^{(7)}$. En el caso de pacientes pediátricos, la hipertrofia de tonsilas y de tejido adenoideo es una causa común de SAOS ${ }^{(14)}$. Además, factores genéticos que influyan en la morfología craneofacial, reduzcan el tamaño de las cavidades nasales alterando el paso de aire, o generen síndromes craneofaciales, también predisponen a sufrir de SAOS. Estos incluyen el retrognatismo mandibular, deficiencia anteroposterior del tercio medio facial, paladar alto y estrecho, mordida abierta anterior, biotipo dólicofacial y posición descendida del hueso hioides ${ }^{(15)}$.

\section{Abordaje diagnóstico}

El Odontólogo debe estar familiarizado con los signos y síntomas de SAOS, permitiéndole hacer un screening en sus pacientes y la derivación oportuna a un médico especialista en medicina del sueño para el diagnóstico definitivo. Es importante llevar a cabo una anamnesis que registre comorbilidades asociadas (hipertensión arterial, cardiopatías, diabetes mellitus tipo 2, dislipidemia, obesidad, etc.) y signos y síntomas comunes, que incluyen ronquido habitual, somnolencia diurna excesiva, despertar abrupto con sensación de ahogo, cefaleas matutinas, dificultad de concentración, entre otros ${ }^{(16)}$. Asimismo, en el contexto Ortodóncico, es necesario realizar un diagnóstico ortodóncico acabado, registrando alteraciones craneofaciales y malos hábitos asociados $^{(15)}$. Es en este contexto en que pueden aplicarse métodos de screening útiles para SAOS. Dentro de los métodos recomendados por la Academia Americana de Medicina del Sueño (AASM), se encuentra validado para adultos el cuestionario STOP-BANG ${ }^{(17)}$, y para niños el Cuestionario Pediátrico del Sueño (PSQ)(18). Ambos cuentan con alta sensibilidad diagnóstica, sobre todo en SAOS moderado a severo.

Respecto a los registros radiográficos que comúnmente se solicitan para ortodoncia, tanto la telerradiografía de perfil como la tomografía computarizada de haz cónico (CBCT) carecen de la capacidad de evaluar la función de la vía aérea. Por tanto, no deben usarse como un método de screening de SAOS ${ }^{(15)}$, aunque sí aportan información valiosa sobre características craneofaciales de riesgo o en la evaluación de estructuras que puedan generar obstrucción.

Por último, el diagnóstico definitivo de apnea obstructiva del sueño debe ser realizado por un médico, utilizando el gold standard, que es una polisomnografía (PSG) nocturna(16). Para cuantificar los eventos obstructivos y la severidad de la apnea obstructiva del sueño, se utiliza el índice de apnea-hipoapnea (AHI), que corresponde a los eventos obstructivos ocurridos durante todo el sueño, dividido por las horas totales de sueño. Así, se obtiene la clasificación de severidad en adultos: leve $(A H I \geq 5 / h,<15 / h)$, moderada $(A H I \geq 15 / h,<30 / h)$ y severa $\left(A H I \geq 30 / h^{(16)}\right.$; y en niños: leve $(A H I \geq 1 / h,<5 / h)$, moderada $(A H I \geq 5 / h$, $<10 / h$ ) y severa $(A H I \geq 10 / h)^{(19)}$. Mayor detalle de los criterios diagnósticos pueden encontrarse en las guías clínicas desarrolladas por la $\mathrm{AASM}^{(16)}$.

\section{Tratamiento}

El tratamiento debe ser interdisciplinario, ya que son múltiples los factores que pueden influir en la etiopatogenia de este trastorno. Al igual como debe ser el médico especialista el que haga el diagnóstico de SAOS, este es quien prescribe y lidera el curso del tratamiento, teniendo en cuenta factores como la severidad, los síntomas y repercusiones en la calidad de vida, las comorbilidades y secuelas presentes, y no menos importante, las preferencias del paciente ${ }^{(4,15)}$. El rol del Ortodoncista, entonces, es trabajar de forma colaborativa y proveer tratamiento ortodóncico, cuando sea necesario y no interfiera con la terapia médica(15).

Dentro de las alternativas terapéuticas en adultos, destaca la terapia con presión positiva en la vía aérea (PAP), los cambios de estilo de vida como la pérdida de peso, los dispositivos de avance mandibular (DAM) y la cirugía de avance maxilomandibular (AMM).

La terapia con PAP es la primera línea de tratamiento del SAOS ${ }^{(16,20)}$, según las recomendaciones de la AASM. Se reportan mejorías en el AHI, disfunción cognitiva, somnolencia y calidad de vida ${ }^{(4,11,21,22)}$. Particularmente en pacientes obesos o con sobrepeso, la pérdida de peso en conjunto a la terapia de CPAP, genera mayores efectos en la disminución del AHI, la resistencia a la insulina, los niveles de colesterol y la presión arterial ${ }^{(11,23-26)}$.

Otra alternativa de tratamiento son los DAM, que son aparatos que permiten mantener la permeabilidad de la vía aérea, gracias a que estabilizan la mandíbula y las estructuras faríngeas, previniendo su colapso durante el sueño ${ }^{(27)}$. Su indicación es en pacientes con SAOS leve-moderado que se resisten a utilizar $\operatorname{CPAP}^{(16,27)}$. Sin embargo, puede considerarse su indicación en casos severos si el paciente no adhiere a la terapia con $\mathrm{CPAP}^{(28)}$, ya que se ha visto que a pesar de que el CPAP tiene mayor efecto en el AHI, debido a su menor adherencia no existen diferencias en las mejorías en somnolencia, función cognitiva y calidad de vida, respecto a los $\operatorname{DAM}^{(28,29)}$.

Por ser una terapia irreversible, la cirugía de avance maxilomandibular por lo general se reserva para casos severos de SAOS, en pacientes adultos con un problema sagital esqueletal subyacente, con indicación ortodóncica, y que no adhieren al tratamiento con CPAP o DAM ${ }^{(15)}$. Un meta-análisis reciente determinó que esta terapia es efectiva en aumentar significativamente el volumen y espacio faríngeo, con obtención de valores AHI bajo $20 / \mathrm{h}^{(30)}$.

Respecto al tratamiento de SAOS en niños, la adenotonsilectomía se considera como el tratamiento estándar en la mayoría de los casos $^{(14)}$, existiendo alto nivel de evidencia sobre los beneficios del tratamiento temprano, sobre todo en preescolares, con mejoras en la función neurocognitiva, coeficiente intelectual, calidad de vida y $\mathrm{AHI}^{(14,31)}$. No obstante, debido a que se han reportado complicaciones postquirúrgicas tempranas, la terapia farmacológica antiinflamatoria parece ser una alternativa prometedora en SAOS leve-moderado( ${ }^{(32,33)}$.

También, terapias ortodóncicas como la expansión maxilar rápida (EMR) cuentan con reportes de larga data sobre sus efectos favorables en la vía aérea ${ }^{(34)}$. Se ha visto que tanto disyuntores de anclaje dentario, 
como de anclaje óseo, tienen efectos esqueletales, con expansión transversal a nivel de la sutura palatina. La diferencia entre el tipo de anclaje, descrita en pacientes adolescentes, es el grado de la apertura sutural y el efecto secundario dentoalveolar ${ }^{(35,36)}$. Así, se ha reportado que en adolescentes la expansión con anclaje óseo puede incrementar la apertura sutural más de 2,5 veces lo logrado con anclaje dentario, observándose que el $68 \%$ de la expansión generada a nivel de primer molar correspondería a la expansión sutural, versus un $26 \%$ en el caso de disyunción con anclaje dentario( ${ }^{(36)}$. Esta apertura sutura se traduce en una expansión del piso y cavidad nasal. Un estudio en pacientes en crecimiento determinó que un promedio de $5,34 \mathrm{~mm}$ de expansión a nivel molar con un disyuntor tipo Hyrax, se traducía en una expansión promedio del piso nasal de 3,05 $\mathrm{mm}^{(37)}$. Es decir, de la expansión transversal a nivel molar, es esperable que a nivel de piso nasal se observe una expansión que corresponde a un $57 \%$ del total. Este efecto a nivel anatómico ha sido respaldado también a nivel funcional, con revisiones sistemáticas y meta-análisis que apoyan el efecto secundario que estas terapias ortodóncicas tendrían en mejorar el nivel de saturación de oxígeno y disminuir significativamente el $\mathrm{AHI}$ en pacientes pediátricos con deficiencia transversal del maxilar y SAOS ${ }^{(38,39)}$. Es importante recalcar, que por ningún motivo esto justifica tratamientos de expansión maxilar en pacientes sin una indicación primariamente ortodóncica ${ }^{(15)}$.

\section{DISCUSIÓN}

Respecto a las alternativas de tratamiento señaladas anteriormente el médico especialista determinará la mejor estrategia de tratamiento prevista para cada caso en particular, teniendo en cuenta las preferencias individuales de cada paciente. Como alternativa más permanente, la cirugía de avance maxilomandibular puede resultar atractiva. Sin embargo, esta tiene un elevado costo económico, riesgos médicos relevantes, y no estaría recomendada en casos con protrusión bimaxilar, por el desmedro estético que tendría para el paciente. Luego, a pesar de ser el estándar de tratamiento, la terapia con CPAP no está exenta de inconvenientes, como es su bajo nivel de adherencia reportada, debido a la incomodidad, inconveniencia, costos y posible claustrofobia que puede generar en el paciente ${ }^{(40)}$

Desde este punto de vista, los DAM han demostrado ser una alternativa de tratamiento muy valiosa. Como ya se mencionó, tienen efectos en la calidad de vida, somnolencia y función cognitiva comparables a los del CPAP, por lo que son recomendados en pacientes que no adhieren a esta primera opción de tratamiento. No obstante lo anterior, muchos profesionales desconocen los efectos adversos que éstos pueden tener en la oclusión. Los efectos son progresivos y significativos, observándose una mayor proinclinación de incisivos inferiores, reducción del overjet y overbite, mordida abierta posterior, y posible desarrollo de mordida invertida ${ }^{(41,42)}$. Estos efectos justifican una supervisión ortodóncica con controles al menos cada 6 meses, siendo el ortodoncista el profesional idóneo para ello(15).

Otro aspecto importante a discutir es la controversia sobre el efecto que tendrían las extracciones dentarias en las dimensiones de la vía aérea superior y su rol en el SAOS. Es relevante analizar críticamente qué es lo que la evidencia disponible dice al respecto, y con ello, evitar la desinformación, sobre todo en el ámbito ortodóncico. Las revisiones sistemáticas disponibles al respecto plantean que la realización de extracciones puede reducir las dimensiones de la orofaringe, la que se daría junto a un descenso del hueso hioides, con gran variabilidad según la etnia ${ }^{(43)}$ y dependiendo de la indicación de extracción, particularmente cuando se realizan para la retracción de incisivos, no así para tratar apiñamiento(44). Sin embargo, los artículos reportados sólo se basan en la medición de dimensiones, sin evaluar la funcionalidad con un estudio de PSG de los sujetos. Así, los autores de esta revisión concluyen que no se ha demostrado que una disminución de la vía aérea se traduzca en que ésta se vuelva más colapsable ${ }^{(44)}$. En línea con lo anterior, se ha aconsejado evitar los tratamientos ortodóncicos con extracciones por el potencial riesgo a desarrollar SAOS. A su vez, se han asociado las agenesias y ausencia de piezas dentarias por otras causas con SAOS aconsejando evitar exodoncias en tratamiento ortodóncicos ${ }^{(45)}$. Sin embargo, vale la pena destacar que dicha recomendación se realizó en base a un estudio observacional en pacientes evaluados en una clínica de sueño con diagnóstico de SAOS, sin grupo control, y sin considerar posibles variables de confusión importantes.

Otra perspectiva es la que ofrece el estudio realizado por Larsen y cols. ${ }^{(46)}$, que representa el estudio más extenso publicado a la fecha al respecto, y que evaluó la prevalencia de SAOS en 2700 pacientes con 4 extracciones de premolares, comparándola a un número similar de individuos sin extracciones, ajustados por edad, sexo e IMC. Su estudio concluyó que la prevalencia de SAOS confirmado mediante PSG, no fue significativamente diferente. Corroborando así, que las extracciones dentales no serían un factor de riesgo para el desarrollo de SAOS(46). Dado lo anterior, resulta razonable realizar indicación de extracciones basado en un diagnóstico ortodóncico exhaustivo, teniendo en consideración variables asociadas a la naturaleza de la maloclusión, así como la vía aérea. Es necesario comprender que cualquier terapia que retruya los incisivos, ya sea por extracción de premolares o por distalización de molares luego de extraer segundos o terceros molares, cae en esta categoría.

Por último, como el nivel de evidencia de los estudios mencionados es de menor calidad que lo desarrollado en el estudio de Larsen y cols., es razonable mantener protocolos terapéuticos que consideren exodoncia de premolares, pero a la vez siempre evaluar su indicación en relación con aspectos conocidos por predisponer a SAOS, como lo son las características demográficas y craneofaciales ya mencionadas.

\section{CONCLUSIÓN}

La apnea obstructiva del sueño es un trastorno crónico multifactorial que produce serias secuelas y afecta gravemente la calidad de vida de aquellos que la padecen. Tanto el odontólogo general, como el especialista tienen un rol importante partiendo por una anamnesis y examen clínico adecuado, haciendo uso de herramientas de screening que se encuentran validadas. Además, durante el tratamiento pueden formar parte de un equipo interdisciplinario, según las múltiples alternativas de tratamiento disponibles vistas en esta revisión.

Estas terapias tienen sus indicaciones, debiendo ser el médico tratante el que lidere el dagnóstico y prescriba el tratamiento, tomando en cuenta varios factores, dentro de los cuales están las preferencias del paciente.

Respecto al rol controversial de extracciones y reducción de vía aérea, en un fenómeno multifactorial como es el del SAOS, reducir la problemática a los volúmenes sin acoplarlos a la función parece poco razonable, sin existir evidencia científica que contraindique la terapia ortodóncica con extracciones de premolares en pacientes con SAOS.

\section{CONFLICTOS DE INTERÉS Y FUENTE DE FINANCIACIÓN}

Los autores no tienen conflictos de interés ni una fuente de financiación para esta publicación.

\section{RELEVANCIA CLÍNICA}

El síndrome de apnea obstructiva del sueño es una patología grave, altamente prevalente y que afecta severamente la calidad y expectativa de vida de quienes la padecen. El odontólogo general y especialista cumplen un rol relevante tanto en su reconocimiento temprano, como en su tratamiento en un contexto interdisciplinario. La siguiente revisión bibliográfica tiene por objetivo describir las casusas, secuelas y métodos de screening, además de discutir las alternativas terapéuticas disponibles y sus controversias, con énfasis en la perspectiva ortodóncica. 


\section{Bibliografía}

1. Kapur VK, Auckley DH, Chowdhuri S, Kuhlmann DC, Mehra R, Ramar K, et al. Clinical practice guideline for diagnostic testing for adult obstructive sleep apnea: an American Academy of Sleep Medicine clinical practice guideline. J Clin Sleep Med. 2017;13(3):479-504.

2. Hla KM, Young T, Hagen EW, Stein JH, Finn LA, Nieto FJ, et al. Coronary hear disease incidence in sleep disordered breathing: the Wisconsin Sleep Cohort Study. Sleep. 2015 May 1;38(5):677-84

3. The top 10 causes of death [Internet]. [consultado 17/12/2019]. Disponible en: https://www.who.int/news-room/fact-sheets/detail/the-top-10-causes-of-death

4. Laratta CR, Ayas NT, Povitz M PS. Diagnosis and treatment of obstructive sleep apnea in adults. Cmaj. 2017;Dec 4;189(48):E1481-8.

5. Franklin KA, Lindberg $E$. Obstructive sleep apnea is a common disorder in the population-A review on the epidemiology of sleep apnea. J Thorac Dis. 2015;7(8):1311-22.

6. Peppard PE, Young T, Barnet JH, Palta M, Hagen EW, Hla KM. Increased prevalence of sleep-disordered breathing in adults. Am J Epidemiol. 2013 May 1;177(9):1006-14.

7. Peppard PE, Hagen EW. The last 25 years of obstructive sleep apnea epidemiology and the next 25? Am J Respir Crit Care Med. 2018;197(3):310-2.

8. Benjafield AV, Ayas NT, Eastwood PR, Heinzer R, Ip MS, Morrell MJ, et al. Estimation of the global prevalence and burden of obstructive sleep apnoea: a literature-based analysis. Lancet Respir Med. 2019 Aug 1;7(8):687-98.

9. Prevalence - Sleep eBook [Internet]. [consultado 14/11/2019]. Disponible en: http://sleepdisorders.sleepfoundation.org/sleep-related-breathing-disorders/ obstructive-sleep-apnea-syndrome/prevalence/

10. Javaheri S, Barbe F, Campos-Rodriguez F, Dempsey JA, Khayat R, Javaheri S, et al. Sleep Apnea: Types, Mechanisms, and Clinical Cardiovascular Consequences. J Am Coll Cardiol. 2017;69(7):841-58

11. Seetho IW, Wilding JP. Obesity and Obstructive Sleep Apnea Syndrome. En: Sbraccia P, Finer N, editor. Obesity: Pathogenesis, Diagnosis, and Treatment. Cham: Springer; 2019. p.243-271.

12. Morsy NE, Farrag NS, Zaki NFW, Badawy AY, Abdelhafez SA, El-Gilany AH, et al. Obstructive sleep apnea: Personal, societal, public health, and legal implications. Rev Environ Health. 2019;34(2):153-69.

13. Shi $Y$, Luo H, Liu H, Hou J, Feng $Y$, Chen J, et al. Related biomarkers of neurocognitive impairment in children with obstructive sleep apnea. Int J Pediatr Otorhinolaryngol. 2019 Jan 1;116:38-42.

14. Venekamp RP, Hearne BJ, Chandrasekharan D, Blackshaw H, Lim J, Schilder AG. Tonsillectomy or adenotonsillectomy versus non-surgical management for obstructive sleep-disordered breathing in children. Cochrane Database Syst Rev. 2015(10) CD011165

15. Behrents RG, Shelgikar AV, Conley RS, Flores-Mir C, Hans M, Levine M, et al. Obstructive sleep apnea and orthodontics: An American Association of Orthodontists White Paper. Am J Orthod Dentofacial Orthop. 2019 Jul 1;156(1):13-28.

16. Epstein LJ, Kristo D, Strollo PJ, Friedman N, Malhotra A, Patil SP, et al. Clinical guideline for the evaluation, management and long-term care of obstructive sleep apnea in adults. J Clin Sleep Med 2009;5(3):263-276

17. Chung F, Yegneswaran B, Liao $P$, Chung $S A$, Vairavanathan $S$, Islam $S$, e al. STOP Questionnaire: A Tool to Screen Patients for Obstructive Sleep Apnea. Anesthesiology. 2008;(108):812-21.

18. Chervin RD, Hedger K, Dillon JE PK. Pediatric sleep questionnaire (PSQ): validity and reliability of scales for sleep-disordered breathing, snoring, sleepiness, and behavioral problems. Sleep Med. 2000;1(1):21-32.

19. Dehlink E, Tan HL. Update on paediatric obstructive sleep apnoea. J Thorac Dis. 2016 Feb;8(2):224

20. Patil SP, Ayappa IA, Caples SM, Kimoff RJ, Patel SR, Harrod CG. Treatment of adult obstructive sleep apnea with positive airway pressure: an American Academy of Sleep Medicine clinical practice guideline. J Clin Sleep Med. 2019;15(2):335-343 21. Schwartz M, Acosta L, Hung YL, Padilla M, Enciso R. Effects of CPAP and mandibular advancement device treatment in obstructive sleep apnea patients: systematic review and meta-analysis. Sleep Breath. 2018 Sep 1;22(3):555-68. 22. Cammaroto G, Galletti C, Galletti F, Galletti B, Galletti C, Gay-Escoda C Mandibular advancement devices vs nasal-continuous positive airway pressure in the treatment of obstructive sleep apnoea. Systematic review and meta-analysis. Med Oral Patol Oral Cir Bucal. 2017 Jul 1;22 (4):e417-24.

23. Toschi-Dias E, Trombetta IC, Silva VJD, Maki-Nunes C, Cepeda FX, Alves MJNN, et al. Diet associated with exercise improves baroreflex control of sympathetic nerve activity in metabolic syndrome and sleep apnea patients. Sleep Breath. 2019;23(1):143-51.

24. Tuomilehto HPI, Seppä JM, Partinen MM, Peltonen M, Gylling H, Tuomilehto
JOI, et al. Lifestyle intervention with weight reduction: First-line treatment in mild obstructive sleep apnea. Am J Respir Crit Care Med. 2009;179(4):320-7.

25. Araghi $\mathrm{MH}$, Chen Y-F, Jagielski A, Choudhury S, Banerjee D, Hussain S, et al. Effectiveness of Lifestyle Interventions on Obstructive Sleep Apnea (OSA): Systematic Review and Meta-Analysis. Sleep. 2013;36(10):1553-62.

26. Joosten SA, Hamilton GS, Naughton MT. Impact of weight loss management in OSA. Chest. 2017 Jul 1;152(1):194-203

27. Ilea A, Timuș D, Höpken J, Andrei V, Băbțan AM, Petrescu NB, et al. Oral appliance therapy in obstructive sleep apnea and snoring-systematic review and new directions of development. Cranio. 2019 Oct 7:1-2.

28. Ramar K, Dort LC, Katz SG, Lettieri CJ, Harrod CG, Thomas SM, et al. Clinical practice guideline for the treatment of obstructive sleep apnea and snoring with oral appliance therapy: an update for 2015: an American Academy of Sleep Medicine and American Academy of Dental Sleep Medicine clinical practice guideline. J Clin Sleep Med. 2015 Jul;11(7):773-827.

29. Cammaroto G, Galletti C, Galletti F, Galletti B, Galletti C, Gay-Escoda C Mandibular advancement devices vs nasal-continuous positive airway pressure in the treatment of obstructive sleep apnoea. Systematic review and meta-analysis. Med Oral Patol Oral Cir Bucal. 2017 Jul 1;22 (4):e417-24.

30. Giralt-Hernando M, Valls-Ontañón A, Guijarro-Martínez R, Masià-Gridilla J, Hernández-Alfaro F. Impact of surgical maxillomandibular advancement upon pharyngeal airway volume and the apnoea-hypopnoea index in the treatment of obstructive sleep apnoea: Systematic review and meta-analysis. BMJ Open Respir Res. 2019;6(1):1-13.

31. Song SA, Tolisano AM, Cable BB, Camacho M. Neurocognitive outcomes after pediatric adenotonsillectomy for obstructive sleep apnea: A systematic review and meta-analysis. Int J Pediatr Otorhinolaryngol. 2016 Apr 1;83:205-10.

32. Tan H-L, Kheirandish-Gozal L, Gozal D. Adenotonsillectomy in Pediatric OSA Time to Look Elsewhere. Curr Sleep Med Reports. 2018;4(3):243-53.

33. Liming BJ, Ryan M, Mack D, Ahmad I, Camacho M. Montelukast and Nasal Corticosteroids to Treat Pediatric Obstructive Sleep Apnea: A Systematic Review and Meta-analysis. J Otolaryngol Head Neck Surg. 2019;160(4):594-602.

34. Haas AJ. Rapid expansion of the maxillary dental arch and nasal cavity by opening the midpalatal suture. Angle Orthod. 1961 Apr;31(2):73-90.

35. Lin L, Ahn HW, Kim SJ, Moon SC, Kim SH, Nelson G. Tooth-borne vs bone-borne rapid maxillary expanders in late adolescence. Angle Orthod. 2015 Mar;85(2):25362.

36. Celenk-Koca T, Erdinc AE, Hazar S, Harris L, English JD, Akyalcin S. Evaluation of miniscrew-supported rapid maxillary expansion in adolescents: a prospective randomized clinical trial. Angle Orthod. 2018 Nov;88(6):702-9.

37. Fastuca $R$, Lorusso $P$, Lagravère $M O$, Michelotti $A$, Portelli $M$, Zecca PA, et al. Digital evaluation of nasal changes induced by rapid maxillary expansion with different anchorage and appliance design. BMC Oral Health. 2017 Dec 1;17(1):113. 38. Machado-Júnior AJ, Zancanella E, Crespo AN. Rapid maxillary expansion and obstructive sleep apnea: A review and meta-analysis. Med Oral Patol Oral Cir Bucal. 2016 Jul;21(4):e465.

39. Camacho M, Chang ET, Song SA, Abdullatif J, Zaghi S, Pirelli P, et al. Rapid maxillary expansion for pediatric obstructive sleep apnea: A systematic review and meta-analysis. Laryngoscope. 2017 Jul;127(7):1712-9.

40. Rotenberg BW, Murariu D, Pang KP. Trends in CPAP adherence over twenty years of data collection: a flattened curve. J Otolaryngol Head Neck Surg. 2016 Dec $1 ; 45(1): 43$

41. Araie T, Okuno K, Minagi HO, Sakai T. Dental and skeletal changes associated with long-term oral appliance use for obstructive sleep apnea: a systematic review and meta-analysis. Sleep Med Rev. 2018 Oct 1;41:161-72.

42. Sato K, Nakajima T. Review of systematic reviews on mandibular advancement oral appliance for obstructive sleep apnea: The importance of long-term follow-up. Jpn Dent Sci Rev. 2020 Dec 1;56(1):32-7.

43. $\mathrm{Ng} \mathrm{JH}$, Song YL, Yap AU. Effects of bicuspid extractions and incisor retraction on upper airway of Asian adults and late adolescents: A systematic review. J Oral Rehabil. 2019 Nov;46(11):1071-87

44. Hu Z, Yin X, Liao J, Zhou C, Yang Z, Zou S. The effect of teeth extraction for orthodontic treatment on the upper airway: a systematic review. Sleep Breath. 2015 May 1;19(2):441-51.

45. Guilleminault C, Abad VC, Chiu HY, Peters B, Quo S. Missing teeth and pediatric obstructive sleep apnea. Sleep Breath. 2016 May 1;20(2):561-8.

46. Larsen AJ, Rindal DB, Hatch JP, Kane S, Asche SE, Carvalho C, et al. Evidence supports no relationship between obstructive sleep apnea and premolar extraction: An electronic health records review. J Clin Sleep Med. 2015;11(12):1443-8. 